Accelerator Division

Alternating Gradient Synchrotron Department

BROOKHAVEN NATIONAL LABORATORY

Upton, New York 11973

Accelerator Division

Technical Note

AGS/AD/Tech. Note No. 354

\title{
MULTITURN INJECTION WITH COUPLING
}

\section{T. Roser}

November 7, 1991

Multiturn injection is possible when the acceptance of the circular accelerator is larger than the emittance of the injected beam. The goal of any multiturn injection scheme is then to fill the ring acceptance evenly with the injected beam. This process is also called "phase space painting." Typically the beam is injected in the horizontal plane with an electrostatic inflector bringing the beam into the ring acceptance. The inflector has to be positioned between the injected beam and the closed orbit as is shown in the space diagram in Fig. 1. The dashed circles in Fig. 1 show the phase space position of the same particles for five consecutive turns for a fractional betatron tune of $n / 5(n=1 \ldots 4)$. Clearly only five turns can be injected in this case. For a smaller emittance of the injected beam and/or larger acceptance more turns can be injected at the appropriate tune value. A detailed description of this scheme is given by J. Wei and S. Y. Lee ${ }^{1}$ and C. Gardner. ${ }^{2}$

This scheme will only fill the horizontal acceptance leaving the vertical acceptance largely empty. In practice, coupling between horizontal and vertical motion will dilute the beam in the vertical phase space until it matches approximately the horizontal emittance. This dilution can be avoided by painting the injected beam not only into the horizontal phase space but also into the vertical phase space. To accomplish this, sufficient coupling between the horizontal and vertical motion has to be present during injection. Using coupling to simultaneously paint both the horizontal and vertical phase space has the potential to significantly increase the number of turns that can be injected into a given acceptance. In principle, this number can be the square of the number that is achievable by injecting into the horizontal plane only. 


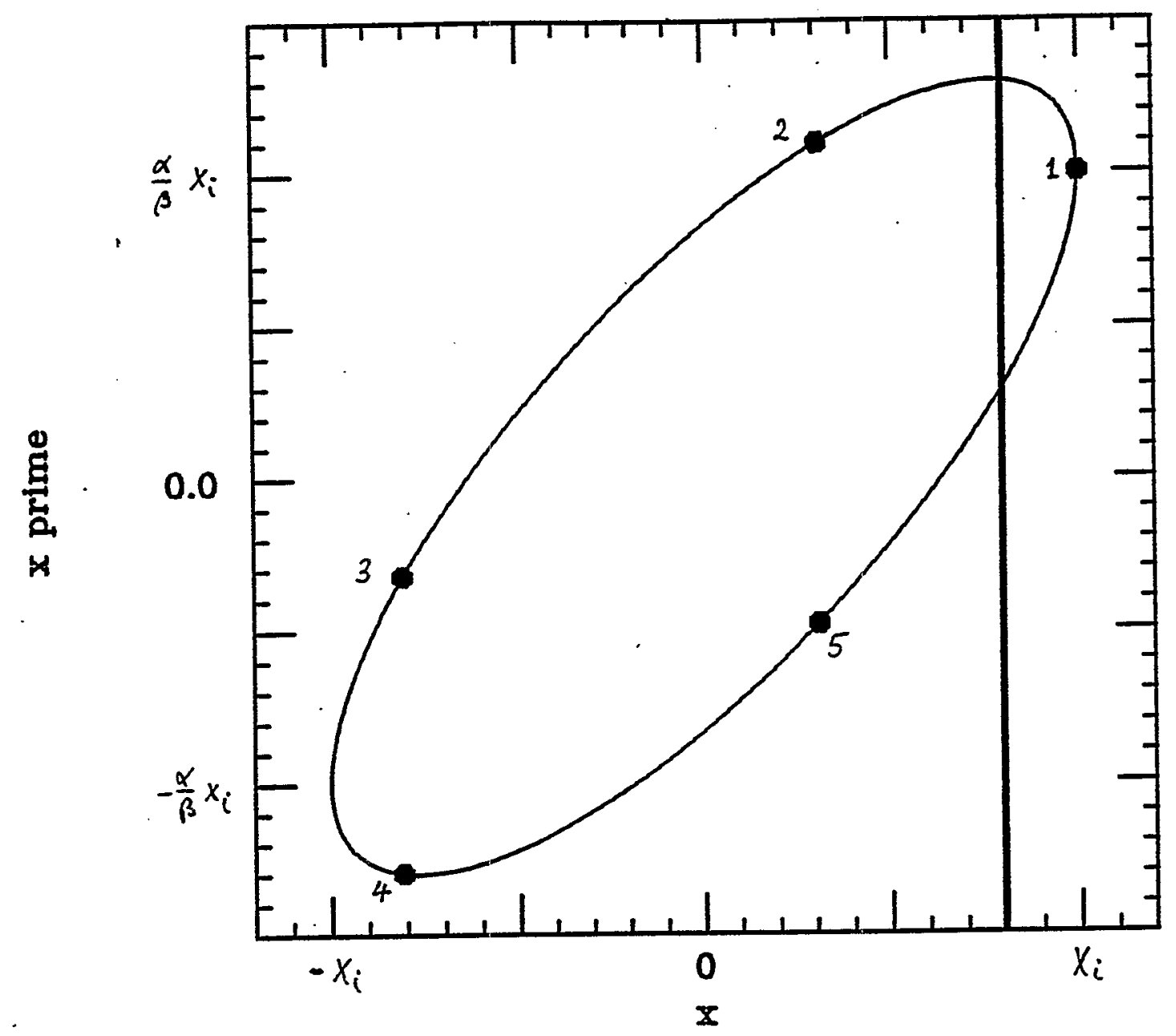

Figure 1.

In the following, I will develop the basic requirements for linear coupling with a simple model consisting of a ring with a single thin skew quadrupole located at injection. The transport matrix for the thin skew quadrupole is

$$
\left(\begin{array}{ll}
\mathbf{1} & \mathrm{Q} \\
\mathrm{Q} & \mathbf{1}
\end{array}\right)
$$

with

$$
Q=\left(\begin{array}{ll}
0 & 0 \\
q & 0
\end{array}\right)
$$

and the rest of the ring is given by 


$$
\left(\begin{array}{cc}
M & 0 \\
0 & N
\end{array}\right)=\left(\begin{array}{cc}
\cos \left(2 \pi \mathrm{Q}_{\mathrm{x}}\right)+\mathrm{J}_{\mathrm{x}} \sin \left(2 \pi \mathrm{Q}_{\mathrm{x}}\right) & 0 \\
0 & \cos \left(2 \pi \mathrm{Q}_{\mathrm{y}}\right)+\mathrm{J}_{\mathrm{y}} \sin \left(2 \pi \mathrm{Q}_{\mathrm{y}}\right)
\end{array}\right)
$$

with

$$
J_{i}=\left(\begin{array}{rr}
\alpha_{i} & \beta_{i} \\
-\gamma_{i} & -\alpha_{i}
\end{array}\right) \quad(i=x, y)
$$

being the standard Courant-Snyder twiss parameter matrix for the uncoupled ring.

I will follow the parametrization of the motion in the 4 dimensional phase space of Edwards and Teng ${ }^{3}$ with some useful extensions and generalizations. ${ }^{4}$ The symplectic one-turn matrix is then

$$
\mathrm{T}=\left(\begin{array}{cc}
\mathbf{M} & \mathbf{n} \\
\mathrm{m} & \mathrm{N}
\end{array}\right)=\left(\begin{array}{cc}
\mathrm{M} & \mathrm{MQ} \\
\mathrm{NQ} & \mathrm{N}
\end{array}\right)
$$

$\mathrm{T}$ is diagonalized by the symplectic rotation matrix $\mathrm{R}$ :

$$
\mathrm{T}=\mathrm{RUR}^{-1}, \quad \mathrm{U}=\left(\begin{array}{cc}
\mathrm{A} & 0 \\
0 & \mathrm{~B}
\end{array}\right), \quad \mathrm{R}=\left(\begin{array}{cc}
\sqrt{1-|\mathrm{E}|} & -\mathrm{SE \tilde {S }} \\
-\mathrm{E} & \sqrt{1-|\mathrm{E}|}
\end{array}\right)
$$

with

$$
S=\left(\begin{array}{rr}
0 & 1 \\
-1 & 0
\end{array}\right)
$$

and

$$
E=-\sqrt{\frac{2}{\Delta_{U}\left(\Delta_{U}-\Delta_{\mathrm{T}}\right)}}(\mathrm{m}-\mathrm{SñS})
$$

$\tilde{E}$ is the transpose of $E,|E|$ is the determinant of $E$, and

$$
\begin{aligned}
& \Delta_{\mathrm{T}}=\operatorname{Tr}(\mathrm{M}-\mathrm{N})=2\left(\cos \left(2 \pi \mathrm{Q}_{\mathrm{x}}\right)-\cos \left(2 \pi \mathrm{Q}_{\mathrm{y}}\right)\right) \\
& \Delta_{\mathrm{U}}=\operatorname{Tr}(\mathrm{A}-\mathrm{B})=2\left(\cos \left(2 \pi \mathrm{Q}_{1}\right)-\cos \left(2 \pi \mathrm{Q}_{2}\right)\right)
\end{aligned}
$$

$Q_{1}$ and $Q_{2}$ are the normal tunes of the diagonalized one-turn matrix $U$. 
When $\Delta_{T}=0$ the effect of the linear coupling by the skew quadrupole is maximized. This means that the original tunes $Q_{x}$ and $Q_{y}$ without coupling are equal. We then have

$$
\Delta_{\mathrm{U}}=\sqrt{\Delta_{\mathrm{T}}^{2}+4 \operatorname{Tr}(\mathrm{mn})+8 \operatorname{lml}}=2 \sin \left(2 \pi \mathrm{Q}_{\mathrm{x}}\right) \sqrt{\operatorname{Tr}\left(\mathrm{J}_{\mathrm{x}} \mathrm{QJ}_{\mathrm{y}} \mathrm{Q}\right)}=2 \mathrm{q} \sin \left(2 \pi \mathrm{Q}_{\mathrm{x}}\right) \sqrt{\beta_{\mathrm{x}} \beta_{\mathrm{y}}}
$$

We can now calculate E:

$$
E=\frac{-1}{\sqrt{2}}\left(\begin{array}{cc}
\sqrt{\frac{\beta_{y}}{\beta_{x}}} & 0 \\
\frac{\alpha_{x}-\alpha_{y}}{\sqrt{\beta_{x} \beta_{y}}} & \sqrt{\frac{\beta_{x}}{\beta_{y}}}
\end{array}\right)
$$

Note that $E$ and thus also the symplectic rotation $R$ are independent of the skew quadrupole strength. This is due to the fact that the uncoupled ring is tuned to the linear coupling resonance $Q_{x}=Q_{y}$. For $R$ we have

$$
R=\frac{1}{\sqrt{2}}\left(\begin{array}{cc:cc}
1 & 0 & -\sqrt{\frac{\beta_{x}}{\beta_{y}}} & 0 \\
0 & 1 & \frac{\alpha_{x}-\alpha_{y}}{\sqrt{\beta_{x} \beta_{y}}} & -\sqrt{\frac{\beta_{y}}{\beta_{x}}} \\
\hdashline \sqrt{\frac{\beta_{y}}{\beta_{x}}} & 0 & 1 & 0 \\
\frac{\alpha_{x}-\alpha_{y}}{\sqrt{\beta_{x} \beta_{y}}} & \sqrt{\frac{\beta_{x}}{\beta_{y}}} & 0 & 1
\end{array}\right)
$$

The symplectic rotation angle $\phi$ introduced by Edwards and Teng is directly related to the determinant of $\mathrm{E}$ :

$$
|E|=\sin ^{2} \phi
$$

In our case we get

$$
\sin ^{2} \phi=\frac{1}{2} \text { or } \phi=45^{\circ} \text {. }
$$

Note, however, that $|E|$ can be negative and therefore $\phi$ is not always defined. 
The twiss parameters of the normal modes are given by the following relations:

$$
\begin{aligned}
\cos \left(2 \pi Q_{1}\right) & =\cos (2 \pi Q)+\frac{1}{2} q \sin (2 \pi Q) \sqrt{\beta_{x} \beta_{y}} \\
\cos \left(2 \pi Q_{2}\right) & =\cos (2 \pi Q)-\frac{1}{2} q \sin (2 \pi Q) \sqrt{\beta_{x} \beta_{y}} \\
\sin \left(2 \pi Q_{1}\right) \alpha_{1} & =\sin (2 \pi Q)\left(\alpha_{x}+\frac{1}{2} q \sqrt{\beta_{x} \beta_{y}}\right) \\
\sin \left(2 \pi Q_{1}\right) \beta_{1} & =\sin (2 \pi Q) \beta_{x} \\
\sin \left(2 \pi Q_{2}\right) \alpha_{2} & =\sin (2 \pi Q)\left(\alpha_{y}-\frac{1}{2} q \sqrt{\beta_{x} \beta_{y}}\right) \\
\sin \left(2 \pi Q_{2}\right) \beta_{2} & =\sin (2 \pi Q) \beta_{y}
\end{aligned}
$$

For a weak skew quadrupole stength $\left(q \sqrt{\beta_{x} \beta_{y}} \ll 1\right)$ these expressions simplify considerably:

$$
\begin{array}{ll}
Q_{1} \approx Q-\frac{1}{2} q \sqrt{\beta_{x} \beta_{y}}, & Q_{2} \approx Q+\frac{1}{2} q \sqrt{\beta_{x} \beta_{y}} \\
\alpha_{1} \approx \alpha_{x}\left(1+q \sqrt{\beta_{x} \beta_{y}}\right), & \alpha_{2} \approx \alpha_{y}\left(1-q \sqrt{\beta_{x} \beta_{y}}\right) \\
\beta_{1} \approx \beta_{x}\left(1+\frac{1}{2} q \sqrt{\beta_{x} \beta_{y}}\right), & \beta_{2} \approx \beta_{y}\left(1-\frac{1}{2} q \sqrt{\beta_{x} \beta_{y}}\right)
\end{array}
$$

To obtain the $\mathrm{x}$ and $\mathrm{y}$ coordinates of the beam motion we transform the time evolution of the normal modes given by

$$
\begin{aligned}
\mathrm{u} & =\sqrt{\omega_{1} \beta_{1}} \cos \psi_{1} \\
\mathrm{p}_{\mathrm{u}} & =-\sqrt{\omega_{1} / \beta_{1}}\left(\sin \psi_{1}+\alpha_{1} \cos \psi_{1}\right) \\
\mathrm{v} & =-\sqrt{\omega_{2} \beta_{2}} \cos \psi_{2} \\
\mathrm{p}_{\mathrm{v}} & =\sqrt{\omega_{2} / \beta_{2}}\left(\sin \psi_{2}+\alpha_{2} \cos \psi_{2}\right),
\end{aligned}
$$

using the symplectic rotation matrix $R$ :

$$
\left(\begin{array}{l}
x \\
p_{x} \\
y \\
p_{y}
\end{array}\right)=R\left(\begin{array}{l}
u \\
p_{u} \\
v \\
p_{v}
\end{array}\right) \text {. }
$$

The invariants $\omega_{1}$ and $\omega_{2}$ and the initial values for the phases $\psi_{1}$ and $\psi_{2}$ are determined by 
the location and direction of the injected beam:

$$
\begin{aligned}
& \omega_{1}=\frac{1}{2 \beta_{1}}\left(x_{i}+\sqrt{\frac{\beta_{x}}{\beta_{y}}} y_{i}\right)^{2}+\frac{\beta_{1}}{2}\left[p_{x}+p_{y} \sqrt{\frac{\beta_{y}}{\beta_{x}}}-\frac{\alpha_{x}-\alpha_{y}}{\sqrt{\beta_{x} \beta_{y}}} y_{i}+\frac{\alpha_{1}}{\beta_{1}}\left(x_{i}+\sqrt{\frac{\beta_{x}}{\beta_{y}}} y_{i}\right)\right]^{2} \\
& \omega_{2}=\frac{1}{2 \beta_{2}}\left(x_{i} \sqrt{\frac{\beta_{y}}{\beta_{x}}}-y_{i}\right)^{2}+\frac{\beta_{2}}{2}\left[p_{x} \sqrt{\frac{\beta_{x}}{\beta_{y}}}-p_{y}+\frac{\alpha_{x}-\alpha_{y}}{\sqrt{\beta_{x} \beta_{y}}} x_{i}+\frac{\alpha_{2}}{\beta_{2}}\left(x_{i} \sqrt{\frac{\beta_{y}}{\beta_{x}}}-y_{i}\right)\right]^{2} .
\end{aligned}
$$

Under the assumption that injection is in the horizontal plane $(\mathrm{y}=0)$ we can then choose $\mathrm{p}_{\mathrm{x}}$ and $\mathrm{p}_{\mathrm{y}}$ to minimize $\omega_{1}$ and $\omega_{2}$ :

$$
\begin{aligned}
& \mathrm{p}_{\mathrm{x}}=-\frac{1}{2}\left(\frac{\alpha_{\mathrm{x}}-\alpha_{\mathrm{y}}}{\beta_{\mathrm{x}}}+\frac{\alpha_{1}}{\beta_{1}}+\frac{\alpha_{2}}{\beta_{2}} \frac{\beta_{\mathrm{y}}}{\beta_{\mathrm{x}}}\right) \mathrm{x}_{\mathrm{i}} \\
& \mathrm{p}_{\mathrm{y}}=-\frac{1}{2}\left(\frac{\alpha_{1}}{\beta_{\mathrm{l}}} \sqrt{\frac{\beta_{\mathrm{x}}}{\beta_{\mathrm{y}}}}-\frac{\alpha_{2}}{\beta_{2}} \sqrt{\frac{\beta_{\mathrm{y}}}{\beta_{\mathrm{x}}}}-\frac{\alpha_{\mathrm{x}}-\alpha_{\mathrm{y}}}{\sqrt{\beta_{\mathrm{x}} \beta_{\mathrm{y}}}}\right) \mathrm{x}_{\mathrm{i}}
\end{aligned}
$$

We then get

$$
\begin{aligned}
& \omega_{1}=\frac{x_{i}^{2}}{21} \\
& \omega_{2}=\frac{x_{i}^{2}}{2 \beta_{2}} \frac{\beta_{y}}{\beta_{x}}
\end{aligned}
$$

The conditions for $p_{x}$ and $p_{y}$ approach the conditions for the uncoupled case in the limit of small skew quadrupole strength:

$$
\begin{array}{ll}
p_{x} \approx-\frac{\alpha_{x}}{\beta_{x}} x_{i}-\frac{\left(\alpha_{x}+\alpha_{y}\right) q}{4} \sqrt{\frac{\beta_{y}}{\beta_{x}}} x_{i} \rightarrow-\frac{\alpha_{x}}{\beta_{x}} x_{i} \\
p_{y} \approx-\frac{\left(\alpha_{x}+\alpha_{y}\right) q}{4} x_{i}
\end{array}
$$

The exact solution for the $\mathrm{x}$ and $\mathrm{y}$ coordinates as a function of time is

$$
\begin{aligned}
& x=\frac{x_{i}}{2}\left(\cos \psi_{1}+\cos \psi_{2}\right)=x_{i} \cos \frac{\psi_{1}-\psi_{2}}{2} \cos \frac{\psi_{1}+\psi_{2}}{2} \\
& y=\frac{x_{i}}{2} \sqrt{\frac{\beta_{y}}{\beta_{x}}}\left(\cos \psi_{1}-\cos \psi_{2}\right)=-x_{i} \sqrt{\frac{\beta_{y}}{\beta_{x}}} \sin \frac{\psi_{1}-\psi_{2}}{2} \sin \frac{\Psi_{1}+\psi_{2}}{2}
\end{aligned}
$$




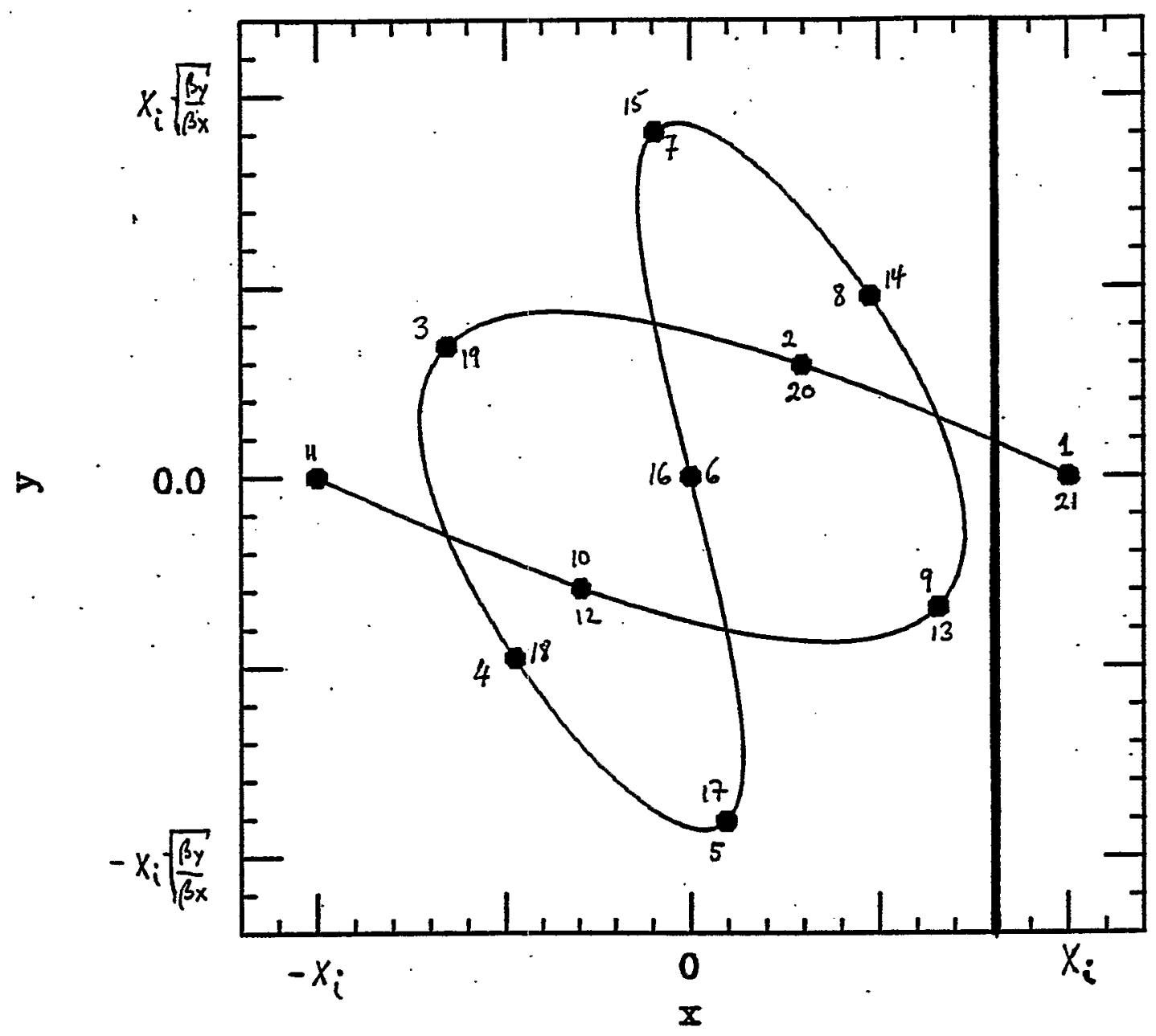

Figure 2 .

Fig. 2 shows the trajectory of the injected beam for

$$
\frac{\Psi_{1}-\Psi_{2}}{\Psi_{1}+\Psi_{2}}=\frac{1}{4}
$$

The dots show the beam locations for $\left(\psi_{1}+\psi_{2}\right) / 2=n / 5(n=1 \ldots 4)$. For this case 20 turns can be injected before the closed orbit has to be moved away from the inflector. This is four times more than for injection without coupling.

For small skew quadrupole strength

$$
\psi_{1}-\psi_{2} \approx 2 \pi q \sqrt{\beta_{x} \beta_{y}} .
$$

For the case described above we then get for the skew quadrupole strength 


$$
\mathrm{q}=\frac{.4}{4} \frac{1}{\sqrt{\beta_{\mathrm{x}} \beta_{\mathrm{y}}}} \cong .011 \mathrm{~m}^{-1}
$$

where we used $\sqrt{\beta_{\mathrm{x}} \beta_{\mathrm{y}}} \approx 9 \mathrm{~m}$. This corresponds to a focal length of $90 \mathrm{~m}$.

After injection is completed the skew quadrupole can be turned off adiabatically. The uncoupled beam then has the following emittances:

$$
\varepsilon_{\mathrm{x}}=\pi \omega_{1}, \quad \varepsilon_{\mathrm{y}}=\pi \omega_{2}
$$

Note that the beam width is smaller than during injection:

$$
\begin{aligned}
& x=\sqrt{\frac{\varepsilon_{x} \beta_{x}}{\pi}}=\sqrt{\frac{x_{i}^{2} \beta_{x}}{2 \beta_{1}}} \approx \frac{x_{i}}{\sqrt{2}} \\
& y=\sqrt{\frac{\varepsilon_{y} \beta_{y}}{\pi}}=\sqrt{\frac{x_{i}^{2} \beta_{y}^{2}}{2 \beta_{2} \beta_{x}}} \approx \frac{x_{i}}{\sqrt{2}} \sqrt{\frac{\beta_{y}}{\beta_{x}}}
\end{aligned}
$$

During injection the surface of the four-dimensional ellipsoid that is defined by $\omega_{1}$ and $\omega_{2}$ will be evenly "painted." To fill also the inside volume of the ellipsoid the distance between the injected beam and the closed orbit can be slowly increased as is proposed for the injection scheme without coupling. In this way it should be possible to inject well over a hundred turns with high efficiency and small final emittance. 
$\therefore \quad:$

\section{References}

1. J. Wei and S. Y. Lee, Simulation of the Multiturn Heavy-Ion Injection at the Booster, Booster Tech. Note 102 (1987).

2. C. Gardner, Multiturn Injection of Heavy Ions into the Booster, Booster Tech. Note 197 (1991).

3. D. A. Edwards and L. C. Teng, Parametrization of Linear Coupled Motion in Periodic Systems, IEEE Trans. on Nucl. Sc. 20, 885 (1973).

4. T. Roser, Generalized Treatment of Linear Motion in Two-Dimensional Periodic Systems, UM-HE 88-8 (1988). 